3. J. S. MacNerney, Stieltjes integrals in linear spaces, Ann. of Math. (2) 61 (1955), 354-367.

4. - Continuous products in linear spaces, J. Elisha Mitchell Sci. Soc. 71 (1955), 185-200.

5. - Determinants of harmonic matrices, Proc. Amer. Math. Soc. 7 (1956), 1044-1046.

6. F. W. Stallard, Differential systems with interface conditions, Oak Ridge National Laboratory Publication no. 1876 (Physics).

7. H. S. Wall, Concerning harmonic matrices, Arch. Math. 5 (1954), 160-167.

Georgia Institute of Technology

\title{
ASYMPTOTIC BEHAVIOR OF THE SOLUTIONS OF NONLINEAR DIFFERENTIAL EQUATIONS
}

T. F. BRIDGLAND, JR.

I. Of considerable significance for the stability analysis of signal transmission systems is the relation between the boundedness and asymptotic behavior of the solutions of the linear differential equation

$$
\frac{d y}{d t}=A(t) y+p(t)
$$

and of the solutions of the nonlinear equation

$$
\frac{d z}{d t}=A(t) z+\phi(z ; t)
$$

Several results on this relationship have been obtained by Perron [1], Bellman [2], Coddington and Levinson [3] and others. The results of the present note-which we state after suitable restriction of (1) and (2)-are further theorems on this relationship.

In (1) and (2) we suppose that the $n \times n$ matrix $A(t)$ has elements which are real-valued, continuous and bounded for $t \geqq 0$ while $p(t)$, $\phi(z ; t)$ are $n$-vectors with the former having elements which are realvalued and continuous for $t \geqq 0$ and the latter having elements which are real-valued and continuous for all $t \geqq 0$ and all $z \in V$, where $V$ is some neighborhood of $z=0$ in the space of $n$-tuples of real numbers.

Received by the editors November 2, 1960 and, in revised form, April 24, 1961. 
Norms of vectors and matrices are denoted by $\|\cdot\|$ and defined as the sum of the moduli of the components. Vectors will be called convergent if their elements tend to finite limits as $t \rightarrow \infty$.

The fundamental matrix of solutions of the homogeneous equation

$$
\frac{d x}{d t}=A(t) x
$$

will be denoted by $X(t)$ where we take $X(0)=I$. We denote by $Y(t)$ the function $\int_{0}^{t} X(t) X^{-1}(\tau) d \tau$ and by $Y(\infty), \lim _{t \rightarrow \infty} Y(t)$ when this limit exists. Our principal result may be stated as

THEOREM 1. If (i) every solution of (1) is convergent for every convergent $p(t)$;

(ii) for sufficiently small $\|z\|, \lim _{t \rightarrow \infty} \phi(z ; t)=\phi(z ; \infty)$;

(iii) for sufficiently small $\beta,\|\phi(0 ; t)\| \leqq \beta$ for $t \geqq 0$;

(iv) for $\epsilon>0$, there exist $\delta>0$ and $T \geqq 0$ such that

$\left\|\phi\left(z_{1} ; t\right)-\phi\left(z_{2} ; t\right)\right\| \leqq \epsilon\left\|z_{1}-z_{2}\right\| \quad$ for $\left\|z_{i}\right\| \leqq \delta, i=1,2$, and $t \geqq T$;

then for every vector $c$ for which $\|c\|$ is sufficiently small a unique bounded solution $z=z(t ; T, c)$ of (2) satisfying $z(T ; T, c)=c$, exists on $[T, \infty)$ and all such solutions converge to the same limit vector, $\xi$, which may be determined uniquely as a solution of the equation

$$
\xi=Y(\infty) \phi(\xi ; \infty) .
$$

II. In [4], it is shown that (i) implies all of the following:

(i') there exist $\alpha>0$ and $K>0$ such that

$$
\left\|X(t) X^{-1}(\tau)\right\| \leqq K \exp [-\alpha(t-\tau)]
$$

for all $t \geqq \tau \geqq 0$;

$\left(\mathrm{i}^{\prime \prime}\right)$ there exists $M>0$ such that $\int_{0}^{t}\left\|X(t) X^{-1}(\tau)\right\| d \tau \leqq M$ for all $t \geqq 0$;

(i'") $\lim _{t \rightarrow \infty} Y(t)$ exists as a matrix with finite elements.

Let us define the successive approximations, $z_{n}(t)$, to the solution of (2) as

$$
\begin{aligned}
\frac{d z_{0}}{d t} & =A(t) z_{0}+\phi\left(z^{*} ; t\right), \quad z_{0}(T)=c ; \\
\frac{d z_{n+1}}{d t} & =A(t) z_{n+1}+\phi\left(z_{n} ; t\right), \quad z_{n+1}(T)=c, \quad n=0,1,2, \cdots,
\end{aligned}
$$

where we assume for the present only that $z^{*}$ is a fixed vector. A solution $z_{n+1}(t)$ of (4) satisfies 


$$
z_{n+1}(t)=X(t) X^{-1}(T) c+\int_{T}^{t} X(t) X^{-1}(\tau) \phi\left(z_{n}(\tau) ; \tau\right) d \tau
$$

from which we obtain, by (iii), (iv), (i') and $\left(\mathrm{i}^{\prime \prime}\right)$, the estimate

$$
\left\|z_{n+1}(t)\right\| \leqq K\|c\|+M \beta+\epsilon M \sup _{T \leqq t}\left\|z_{n}(t)\right\| \text {. }
$$

If for some fixed $\lambda, 0 \leqq \lambda<1$, we suppose $\beta \leqq \lambda(1-\epsilon M) \delta / M$ and $\|c\|<(1-\lambda)(1-\epsilon M) \delta / K$ and take $\epsilon \leqq \mu M^{-1}$ for fixed $\mu, 0<\mu<1$, it follows by (6) that $\sup _{T_{\leq}}\left\|z_{n+1}(t)\right\|<\delta$ if $\sup _{T_{\leq} t}\left\|z_{n}(t)\right\|<\delta$. An estimate similar to (6) shows that when $\left\|z^{*}\right\|<\delta$ then $\sup _{T \leqq t}\left\|z_{0}(t)\right\|<\delta$. This completes the induction and shows that our approximations are bounded on $[T, \infty)$, uniformly for $n=0,1,2, \cdots$.

From (5) we find that

$$
\left\|z_{n+1}(t)-z_{n}(t)\right\| \leqq \mu \sup _{T \leqq \tau \leqq \imath}\left\|z_{n}(\tau)-z_{n-1}(\tau)\right\| ;
$$

hence

$$
\sup _{T \leqq \tau \leqq t}\left\|z_{n+1}(\tau)-z_{n}(\tau)\right\| \leqq \mu \sup _{T \leqq \tau \leqq \tau}\left\|z_{n}(\tau)-z_{n-1}(\tau)\right\|
$$

so that the series $\sum_{n=0}^{\infty} \sup _{T \leq \tau \leq t}\left\|z_{n+1}(\tau)-z_{n}(\tau)\right\|$ converges uniformly for $T \leqq t$. This in turn implies that the $z_{n+1}(t)$ converge to a limit vector $z(t)$ uniformly for $T \leqq t$. From (5) it then follows that $z(t)$ is the unique solution of (2) satisfying

$$
z(t)=X(t) X^{-1}(T) c+\int_{T}^{t} X(t) X^{-1}(\tau) \phi(z(\tau) ; \tau) d \tau .
$$

Now consider the transformation $R$ defined on $\{z \mid\|z\| \leqq \delta\}$ by

$$
R z=Y(\infty) \phi(z ; \infty)
$$

by (iii),

$$
\|R z\| \leqq\|Y(\infty)\|(\epsilon \delta+\beta) \leqq M \epsilon \delta+\lambda(1-\epsilon M) \delta<(1-\lambda) \delta+\lambda \delta=\delta,
$$
and by (iv),

$$
\left\|R z_{1}-R z_{2}\right\| \leqq\|Y(\infty)\| \epsilon\left\|z_{1}-z_{2}\right\| \leqq \mu\left\|z_{1}-z_{2}\right\| .
$$

Since $\mu<1$, Banach's fixed point theorem implies that there exists a unique $\xi,\|\xi\|<\delta$, such that

$$
\xi=R \xi \text {. }
$$

Again from (5) and (iv) we find that 


$$
\left\|z_{n+1}(t)-z_{n}(t)\right\| \leqq \epsilon \int_{T}^{t}\left\|X(t) X^{-1}(\tau)\right\|\left\|z_{n}(\tau)-z_{n-1}(\tau)\right\| d \tau
$$

using $\left(i^{\prime}\right),\left(i^{\prime \prime}\right)$, an argument similar to that for the sufficiency of $[4$, Theorem 1] shows that if $\left\|z_{n}(t)-z_{n-1}(t)\right\| \rightarrow 0$ as $t \rightarrow \infty$ then the integral on the right of (9) tends to zero as $t \rightarrow \infty$. Now from (i), (ii), (4) and [4, Theorem 1] it follows that $\lim _{t \rightarrow \infty} z_{0}(t)=Y(\infty) \phi\left(z^{*} ; \infty\right)$; on the other hand, from (4), (5) and (iv) we have

$$
\left\|z_{1}(t)-z_{0}(t)\right\| \leqq \epsilon \int_{T}^{t}\left\|X(t) X^{-1}(\tau)\right\|\left\|z_{0}(\tau)-z^{*}\right\| d \tau .
$$

Thus, if $z^{*} \equiv \xi$, the integral on the right tends to zero as $t \rightarrow \infty$; this, together with the argument from (9), implies inductively that $\lim _{t \rightarrow \infty} z_{n}(t)=\xi, n=0,1,2, \cdots$. Although this convergence is not necessarily uniform in $n$, the convergence of $z_{n}(t)$ to $z(t)$ is, as noted previously, uniform in $t$; invoking the Moore-Osgood theorem on limits we conlude finally that

$$
\lim _{t \rightarrow \infty} z(t)=\lim _{t \rightarrow \infty} z_{n}(t)=\xi .
$$

REMARK. The simplest way to ensure that (i) is satisfied is to take $A(t)=A+B(t)$ where the characteristic roots of $A$ have only negative real parts and where $\|B(t)\| \rightarrow 0$ as $t \rightarrow \infty$; in this case $Y(\infty)=-A^{-1}$ [4]. One of the simplest functions satisfying (ii), (iii), (iv), is $\phi(z ; t)$ $=f(z)+g(t)$ where

(a) the bound of $\|g(t)\|$ is sufficiently small and $\lim _{t \rightarrow \infty} g(t)$ exists;

(b) $f(0)=0$ and, for $\epsilon>0$, there exists $\delta>0$ such that

$$
\left\|f\left(z_{1}\right)-f\left(z_{2}\right)\right\| \leqq \epsilon\left\|z_{1}-z_{2}\right\| \quad \text { for }\left\|z_{i}\right\| \leqq \delta, i=1,2 .
$$

III. The next theorem shows that the existence of a fixed point of the transformation $R(z) \equiv Y(\infty) \phi(z ; \infty)$ is necessary for the existence of a limit for a solution of (2).

THEOREM 2. If conditions (i) and (ii) of Theorem 1 are satisfied, with the convergence in (ii) uniform in $z$, and if $z(t)$ is a bounded solution of (2) for which $\lim _{t \rightarrow \infty} z(t)=z^{*}$, then $z^{*}$ satisfies $z^{*}=Y(\infty) \phi\left(z^{*} ; \infty\right)$.

For we have

$$
\begin{aligned}
Y(\infty) \phi\left(z^{*} ; \infty\right)-z^{*}= & {\left[z(t)-z^{*}\right]-X(t) X^{-1}(T) c } \\
& -\left[\int_{T}^{t} X(t) X^{-1}(\tau) d \tau-Y(\infty)\right] \phi\left(z^{*} ; \infty\right) \\
& -\int_{T}^{t} X(t) X^{-1}(\tau)\left[\phi(z(\tau) ; \tau)-\phi\left(z^{*} ; \infty\right)\right] d \tau .
\end{aligned}
$$


By hypothesis, the first three terms on the right of (10) tend to zero as $t \rightarrow \infty$. The hypotheses, together with the Moore-Osgood theorem, imply that $\left\|\phi(z(t) ; t)-\phi\left(z^{*} ; \infty\right)\right\|$ tends to zero as $t \rightarrow \infty$; an argument like that of [4] mentioned above then shows that the fourth term on the right of (10) tends to zero. Hence $\left\|Y(\infty) \phi\left(z^{*} ; \infty\right)-z^{*}\right\|<\epsilon$ for every $\epsilon>0$, which proves the theorem.

In the event that, for some $z_{1}, \phi\left(z_{1} ; \infty\right)=0$, then we may dispense with the existence of $Y(\infty)$; to be precise we have

THEOREM 3. If $(\mathrm{v})$ every solution of $(1)$ is bounded for every bounded $p(t)$

(vi) for some $z_{1}$, with $\left\|z_{1}\right\|$ sufficiently small, $\lim _{t \rightarrow \infty} \phi\left(z_{1} ; t\right)=0$;

(vii) for $\epsilon>0$, there exist $\delta>0$ and $T \geqq 0$ such that

$$
\left\|\phi(z ; t)-\phi\left(z_{1} ; t\right)\right\| \leqq \epsilon\|z\| \quad \text { for }\|z\| \leqq \delta \text { and } t \geqq T ;
$$

then for every vector $c$ for which $\|c\|$ is sufficiently small, a bounded solution, $z(t ; T, c)$, of (2) satisfying $z(T ; T, c)=c$ exists on $[T, \infty)$ and all such solutions tend to zero as $t \rightarrow \infty$.

As is well known (vide [4]), (v) implies ( $\left.i^{\prime}\right)$ so that Theorem 3 is in essence a special case of the theorem of $[3$, p. 327] cited previously and in turn implies the result of Bellman in [2].

\section{REFERENCES}

1. O. Perron, Die Stabilitätsfrage bei Differentialgleichungen, Math. Z. 32 (1930) 703-728.

2. R. Bellman, On an application of a Banach-Steinhaus theorem to the study of the boundedness of solutions of nonlinear differential and difference equations, Ann. of Math. 49 (1948), 515-522.

3. E. A. Coddington and N. Levinson, Theory of ordinary differential equations, McGraw-Hill, New York, 1955.

4. T. F. Bridgland, Jr., Asymptotic behavior of the solutions of nonhomogeneous differential equations, Proc. Amer. Math. Soc. 12 (1960), 546-552.

RADIATION INCORPORATED 